\title{
RELATOS Y REFLEXIONES EN TALLERES DEL CONOCIMIENTO
}

\author{
INGER HELEN ERSTAD ${ }^{1}$
}

Directora del proyecto piloto "Stories enhancing Skills". Noruega.

\section{INTRODUCCIÓN}

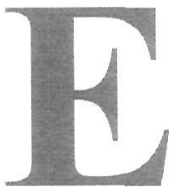

$\mathrm{n}$ este artículo se presenta la base para un proyecto piloto de Leonardo da Vinci, llamado "Stories enhancing Skills" (inglés), "Conocimientos profesionales y relatos en el trabajo social con menores" (español).

El proyecto se va a realizar con base en el conocimiento y la metodología usados en un trabajo de doctorado (Erstad, 2005) de la Universidad de Tromsø en Noruega. En este trabajo, se utilizó un "taller de conocimientos" como herramienta para descubrir y analizar la práctica de los trabajadores sociales, sus experiencias y su quehacer en la actividad profesional en el campo de menores. El contenido del trabajo se adscribe a la tradición del conocimiento práctico que se remonta hasta la antigüedad y Aristóteles (Erstad, 2002).

El taller del conocimiento con sus relatos y sus reflexiones sobre la experiencia va a ser probado como método dentro de los contenidos educativos en la formación postgrado y en la formación continuada en los países nórdicos y en España. La experiencia se quiere ampliar también para Estlandia (países bálticos). El proyecto será evaluado por un equipo externo al grupo de trabajo.

En el trabajo social es necesario buscar métodos basados en conocimientos teóricos estandarizados. La práctica profesional debe basarse en métodos con efectos documentados; a su vez, es de suma importancia tener en cuenta la experiencia de los profesionales del trabajo social y la experiencia de los usuarios de los servicios sociales, para, de este modo, poder afrontar la realidad diaria y las situaciones cambiantes que surgen. Los trabajadores sociales deben desarrollar su juicio profesional con las distintas formas de conocimientos que forman un todo en la práctica profesional. Esta competencia es el tema de los talleres del conocimiento.

\section{LOS RELATOS COMO FUENTE DE CONOCIMIENTOS}

Los relatos se han caracterizado por ser una fuente de conocimientos y comprensión en todos los tiempos y en las distintas culturas. Podríamos decir

I Traducción de Angélica Majos Trepat, coordinadora responsable del proyecto en España. 
que el relato es una forma ancestral que ha acompañado siempre al hombre . Nergård (2000), haciendo referencia a las tradiciones laponas, expone el relato verbal como lugar de acumulación y guarda de conocimientos, que tiene como finalidad el ser un dispositivo para la práctica social.

En la ganadería de los renos, los relatos sobre el terreno son importantes. Los relatos guardan un conocimiento del que los ganaderos no podrían prescindir. En estos relatos, los conocimientos y la experiencia en cuanto a la naturaleza del terreno son almacenados. Este conocimiento se traspasa a las nuevas generaciones, de modo que aprenden relatos directamente relacionados con sitios específicos en el terreno donde trabajan. Los relatos no sólo guardan la experiencia, sino que también "crean" terreno o descubren sitios apropiados para la ganadería.

Los relatos se refieren a zonas concretas y también crean lugares en el terreno, que no existirian en la conciencia sin ellos. Indican lugares peligrosos o lugares que tienen buenos o malos pastos. De este modo, constituyen una concentración especial sobre un lugar o una zona concreta. Podemos llamar a este tipo de relatos "guías" o "guardianes del camino".

La descripción de Nergård de los relatos del terreno y su función es para mí una descripción que recoge la esencia del conocimiento de la experiencia práctica. Son paisajes, terrenos y lugares que crean muchas asociaciones y que pueden ser utilizados como metáforas, incluso en la actividad del campo del trabajo social. Claro está que existen diferencias entre la ganadería de renos y el trabajo social en el campo del menor... Dejémoslo así de momento...

Esta introducción sobre la ganadería de renos se adapta mejor a las condiciones noruegas que a las de España, aunque el significado de la función de los relatos como transmisores de conocimientos se puede reconocer en las distintas tradiciones artesanales y profesionales. Un ejemplo de esta transmisión en España podría ser la sabiduría que existe en los relatos sobre los métodos de los cuidados de los viñedos y sus terrenos de los que depende la calidad del producto final del vino.

\section{EL TALLER DE CONOCIMIENTOS COMO LUGAR DE RE- FLEXIÓN}

También yo utilizo los relatos para las situaciones en la práctica y he profundizado en ello en mi proyecto para la comprensión del profesional del trabajo social y su conocimiento desde la experiencia. Junto con un grupo de trabajadores sociales que ejercen su actividad en el campo del menor, he realizado actividades para desarrollar un acercamiento metódico para recopilar este tipo de conocimientos desde la experiencia. Creamos un espacio de encuentros -un taller de conocimientos-. Hicimos seis reuniones diarias durante un año y medio y en este tiempo dialogamos, trabajando con el conocimiento de la experiencia, y elaboramos un contenido que podemos llamar el conocimiento de la actuación o conocimiento práctico.

Hemos enfocado en las situaciones problemáticas y los dilemas. Los trabajadores sociales contaron sus historias y reflexionamos sobre ellas. Luego, he 
reescrito los relatos, primero como textos sencillos y, más tarde, como ensayos, introduciendo aspectos de Filosofia, Literatura y Ética para discutir los temas que aparecen en los mismos. Cuando era oportuno, añadimos también otros medios, como películas, y otras expresiones artísticas. Todo ello nos ofreció las bases para hacer nuevas reflexiones.

En el proyecto, he utilizado un método de acercamiento al conocimiento práctico que Janik (1996) expone en su libro El concepto del conocimiento en la filosofia práctica. Es la tradición de Aristóteles lo que el autor refleja en su trabajo.

Las preguntas que hago, desde este marco de referencia en cuanto al relato, son, en primer lugar, qué tipo de valoración se hace en las situaciones concretas expuestas. Aquí detenemos un momento la secuencia donde aparece una situación clave del relato. ¿Qué es lo que vio el trabajador social? ¿Qué es lo que comprendió y cómo pudo observarlo? ¿Qué dijo o hizo concretamente? Aquí se necesita volver varias veces sobre el tema para profundizar más.

Llegamos de este modo a aclarar qué es lo que ocurrió y en qué marco actuó el trabajador social. ¿Cuál era el escenario directo y qué podemos suponer que hay detrás? ¿Qué aspectos podrían encontrarse escondidos o fuera de nuestra vista?

Al cambiar el escenario anterior y el de atrás podemos ver más claro las distintas variables de la situación, la cual se abre ante nosotros y nos brinda la posibilidad de usar más elementos de análisis. De este modo, aparece una reflexión que puede abrir y ampliar la perspectiva. Más adelante, podemos preguntarnos cuáles son los aspectos que aparecen como importantes y qué es lo que nos lleva a profundizar más en la comprensión.

Qué nos parece importante y qué menos. Cuál es la semejanza en los relatos y qué es lo diferente en lo que parece casi igual. Qué aparece como análogo y cómo podemos llegar a un conocimiento más profundo de las estructuras básicas.

La tarea de traer a la luz el conocimiento práctico se puede describir como un camino que lleva a descubrir principios implícitos o básicos. El conocimiento que hemos obtenido a través de la reflexión se puede utilizar para formar y reformar ejemplos que pueden llegar a ser una forma de guías. Ello es el camino hacia la ejemplificación.

Si tratamos de encontrar lo que llamaríamos la naturaleza del taller de los conocimientos podríamos decir que es la reflexión continuada que alterna entre el relato y el relato transformado. El relato repetido es la estructura básica que puede ser recreado en distintas formas. Podríamos utilizar el prisma como metáfora. Circulamos alrededor del tema de los relatos, en vez de perseguir un pensamiento estricto y lógico. El taller de los conocimientos aparece como un prisma, donde, de un mismo rayo de luz, pueden salir fracciones que apuntan en distintas direcciones y van más allá de las distintas tradiciones profesionales. Aquí, observamos también la semejanza con la naturaleza del ensayo.

Al usar el prisma como metáfora, ponemos énfasis en el sentido de la vista. Si buscamos una métafora del sentido del oído, la fórmula de taller de música 
sería la más apropiada como analogía para la dinámica y la colaboración en un taller de conocimientos. Lo importante en este sentido sería "tener oído" o prestar el oído al escuchar los relatos y las reflexiones para distinguir entre la armonía, las consonancias y las disonancias.

\section{ESCUCHAR CON ATENCIÓN ES UNA EXIGENCIA: UN EJEMPLO DE GUARDA DEL CAMINO}

En un taller del conocimiento experimenté un reto personal en cuanto a mi destreza de poder escuchar con atención. Yo era la que transcribía los relatos. Aquí, me tocó transformar el relato verbal en un texto escrito del cual íbamos a seguir trabajando. Bastante fácil, pensé, pero surgieron varias reacciones.

Dijeron algunos que se reconocía el relato, pero lo encontraron extraño al leerlo. Una dice: "cuando yo cuento, tengo las impresiones visuales de las distintas situaciones y puedo sentir la atmósfera que habia. Hay tantas cosas en el relato que no aparecen sólo con palabras. Me identifico.mucho más con el relato cuando lo cuento yo" -cuando conté el relato salió de mí, mientras que leyéndolo se presenta ante mí-.

También ha sido un reto transcribir los relatos de la versión verbal de un dialecto noruego al idioma oficial, porque hubo detalles del significado que no logré captar. Sin embargo, la reacción más fuerte de los participantes del taller al leer mi transcripción fueron sus propias expresiones al verlos impresos. " $E l$ relato parece tan sencillo -decía una, refiriéndose a la expresión-, a lo mejor no tiene suficiente valor profesional". Poco a poco, trabajando con los relatos, haciendo reflexiones teóricas y ensayos, la valoración del contenido del relato cambió. "Obtienes tantas cosas de los relatos... -dijeron-".

Durante el proceso, despertó el interés por el paralelismo entre la transcripción y la tarea de escribir historias en su trabajo y lo difícil que puede ser reflejar lo que piensan los clientes:

"Nosotros que contamos las historias aqui sabemos formularlas. No todos los que encontramos saben explicar con palabras lo que piensan y sienten en su situación. No es fácil expresarse con personas desconocidas. Me pregunto muchas veces si lo que escribo es correcto: ¿era eso o lo otro lo que quería decir? Es un deber que los usuarios puedan ver lo que hemos escrito y que estén de acuerdo. En la práctica, tenemos que actuar tan rápido que nos falta el tiempo para ello".

Otro dice, que cuando nosotros, los trabajadores sociales, podemos reaccionar así cuando vemos nuestros relatos por escrito, cómo será la reacción de los clientes cuando ven su vida reffejada por escrito, usando otras palabras y sentidos de los que ellos hubieran usado. Los apuntes de historias sociales son frecuentemente usados como base para valorar los casos en la Fiscalía de menores y, cuando el caso llega allí, puede que haya mucho que perder. Un ejemplo:

"Se trataba de un caso de guarda y tutela temporal voluntaria de un menor. En los documentos se decía que la madre se sentía inútil como 
madre. La madre quería que se borrara esta frase y quería que la entidad de protección de menores le diera una excusa. Se trataba de que ella se encontraba demasiado cansada para poder cuidar a su niño, no tenía que ver con incapacidad. Ella no podía dejar de pensar en lo que habian escrito en este documento".

Aquí podemos ver cómo la expresión idiomática puede definir, explicar o señalar. El hecho de estar demasiado cansada para poder atender a un hijo es una valoración responsable por parte de una madre. La guarda y tutela temporal puede ser justificada por este hecho. Es otra cosa, o a lo mejor, lo contrario, sentirse como una madre inútil, o ser una mala madre. Qué fue lo que dijo realmente en esta situación no lo sabemos, pero la intención fue otra de lo que se había expresado por escrito. Tampoco es difícil imaginar que la expresión "estar demasiado cansada" es un concepto que se puede aceptar mejor que el "sentirse una mala madre" o "una madre incapaz". Aquí, si el trabajador social hubiera sido más sensible colaborando con la madre en cuanto a formular este problema, la situación en sí, de formalizar una tutela temporal, se hubiera llevado a cabo con más aceptación y, a su vez, hubiera facilitado la colaboración con ella, todo en bien del niño. En este caso, tanto ante los ojos de la misma madre como ante los de los otros, la madre sería la que tomaba la responsabilidad del hecho de que se encontraba en una situación de abatimiento y demasiado cansada para cuidar de su hijo. La madre hubiera guardado más su dignidad. En otras palabras, esto tiene que ver también en cómo la madre siente que las autoridades la valoran a ella. De este modo, los cuidados de los detalles y los ojos que ven hacen la gran diferencia entre lo que podríamos llamar sabiduría personal o lo contrario, la falta de ella. Ello no quiere decir necesariamente que se están haciendo las cosas así por maldad o por una falta de ética. Más bien puede deberse a falta de sensibilidad o falta de oído de la otra persona.

\section{EN EL TRABAJO SOCIAL CON EL MENOR SE NECESITA UN ALMACÉN DE RELATOS DEL TERRENO}

En el trabajo social actual con el menor, se usan muchos y distintos conocimientos, los cuales se amplían cada vez más, facilitando de este modo el trabajo. En esta actividad, el reto consiste en saber elegir qué tipo de conocimiento aplicar y utilizar el terreno donde uno se encuentra en cada momento.

En la situación concreta es donde el conocimiento teórico nos dice el qué y el porqué; las habilidades enseñan el cómo estos se unen con el conocimiento de la experiencia de una forma flexible capaz de decidir cuándo es sabio hacer una cosa u otra.

La actividad de campo del trabajo social con el menor tiene una naturaleza de tipo social y práctico. Hay que dialogar con el niño y su familia y las distintas redes sociales para entender lo específico del lugar y lo que se requiere de él. Para poder hacer un juicio o tener una comprensión sabia, se necesita un almacén de relatos, para reconocer y distinguir entre lugares, tanto literal como metafóricamente entendido. Este tipo de entendimiento de cómo acercarse a una situación de una forma útil, se puede también describir como trabajo social 
contextual en el campo del menor (Sau,s 2003) o conocimiento móvil de la actuación (Lykkeslet, 2003).

Los relatos del terreno deben ser desarrollados de manera que aparezcan como algo más que las experiencias de un solo profesional y necesitan ser recopilados y guardados en su forma escrita. En mi trabajo doctoral he profundizado en unos pocos relatos. Para poder comprender lo cambiante del trabajo se necesita un cúmulo de relatos. Mediante el estudio de las distintas situaciones aparecerán múltiples variaciones.

Estas variaciones nos ayudan a obtener una visión nítida para las diferentes facetas y, a través de relatos contrastantes, encontrarnos con que, en situaciones aparentemente iguales, puede haber diferencias. De este modo, podemos discutir cómo hasta el más perspicaz, con su vista aguda -según la terminología de Melø (1979)-, también debe de tener un enfoque abierto y buscador, para evitar lo contrario, que sería una visión borrosa u obsoleta.

En NOU 2000:12 (propuesta de actuación del gobierno noruego en materia del menor), se plantean retos similares. Aquí se dice que es necesario usar el juicio profesional a la vez que hay aspectos problemáticos en todo juicio. "Cuando se utiliza el juicio profesional, en los casos de menores, se puede generar una visión limitada del caso asi como que se puede ampliar la misma visión en el proceso de las decisiones". Opino que la visión limitada se puede evitar utilizando la reflexión y los relatos.

En el trabajo con el menor, se necesitan relatos sobre maneras sabias de actuar que nos puedan enseñar el camino a través de un terreno difícil y lleno de tensiones. Este tipo de relatos tienen la función de ser guías que pueden dar seguridad en las actuaciones para los que vienen detrás y dar esperanzas de que es posible encontrar caminos en el terreno más hostil. Otros relatos nos cuentan dilemas y contrariedades que ocurren en la actividad de la Administración y de los profesionales que trabajan con el menor. Estos relatos nos pueden indicar caminos para actuar en casos difíciles o dilemas que queden abiertos para la reflexión.

También es importante sacar a la luz relatos de casos donde no se vio realmente la situación, de modo que el juicio profesional falló. Es aquí donde el cambio de visión del relato lleva a otra comprensión. Lo experimentado se entiende y lleva a otro marco de entendimiento. Este tipo de relatos abren nuevos caminos en el terreno de la práctica, indicándonos dónde debemos tener precaución cuando nos encontramos en un terreno difícil.

Es una ventaja que la forma del relato sobre el terreno sea corto y con enfoque, tal como en este ejemplo:

"En este caso, la madre de la familia acogedora consiguió que la madre biológica tomara contacto con el médico que trataba al niño y su profesor en el colegio para, de este modo, tener más información sobre su hijo. La madre biológica tiene una actitud crítica ante cualquier actuación y, de este modo, la madre acogedora se libera de ser la persona intermediaria. La madre puede discutir con el médico y el profesor del niño, a la vez que se involucra en el desarrollo de su hijo y puede actuar en su papel de madre". 
El ejemplo tiene un contenido mucho más amplio de lo que podemos leer en estas pocas frases escritas. Para poder beneficiarse de los relatos, como dispositivo profesional de su propia práctica, el profesional debe relacionar las historias con la propia experiencia, trabajando en conciencia, utilizando la asociación de ideas, buscando las estructuras semi-escondidas.

Con Molander (1993), podemos decir que los textos pueden ser más o menos abiertos y que la comprensión surge en un espacio entre el texto y el lector. Los relatos escritos, a diferencia de los verbales, tienen la ventaja de llegar a varios receptores a la vez. Si estos textos son considerados conocimientos en el camino, se podrá añadir un cúmulo de relatos y reflexiones observando la relación entre las normas y lo que es diferente a ellas.

De este modo, los relatos serán objeto de discusión y pueden ser utilizados para seguir desarrollando la práctica. Pueden ser utilizados como ejemplos para nuevos conocimientos y ayudar al profesional a ver lo singular de la situación. La persona experimentada reconocerá la sabiduría de la actuación, mientras que la que tenga menos experiencia puede necesitar más "pistas" que enseñen la profundidad que este ejemplo transmite.

En la profesión de Enfermería, entre otras, se ha trabajado para desarrollar patrones de ejemplo. Su naturaleza es del tipo indicadores. Éstos pueden ser importantes pero no son suficientes y pueden llegar a cubrir diferencias importantes.

El material de los talleres del conocimiento contiene muchos relatos donde ocurren importantes cambios en la experiencia. En estos cambios, ha habido nueva comprensión como resultado del diálogo con la familia y las redes de apoyo, del modo que la experiencia de aquéllos también se ha tenido en cuenta como válida. Lo que antes se había escondido a la vista como "escenario de fondo" ha aparecido y se ha podido ver.

Uno de los relatos se podría describir como un paradigma de cambio de la manera de ver los posibles recursos en la familia extensa. Lo que se puede observar en las reflexiones es el hecho de que este cambio ofrece la posibilidad de obtener nueva comprensión. El hecho de "entender la situación sin más" oculta lo nuevo y frena la posibilidad de ver los detalles.

Otras veces, la mirada es más abierta, como en el caso del profesional que descubre, en un instante, que la definición de los otros puede ser bastante diferente a la suya.

"Estuve haciendo una visita de seguimiento en la Oficina del Menor: El niño estaba en casa y estuvimos charlando sentados en el sofá. El niño me contó que su abuela le había olvidado en una cafetería. Yo le estaba escuchando hasta que la madre le dijo con voz decidida: "con esta señora no debes hablar, recuerda que ella apunta todo lo que dices. Ello puede perjudicar a la abuela». A continuación, yo pensé: "qué tonta e ignorante he sido, pensando que ellos tenian confianza en mì".

Este ejemplo no ofrece indicaciones de actuación, pero sugiere que puede haber situaciones complejas y dilemas entre las funciones de ayuda y control. 
La práctica en el trabajo con el menor está llena de situaciones complejas que se entienden como dilemas. Uno de los participantes en los talleres del conocimiento dijo que "era una satisfacción poder extender los problemas y literalmente "colgarlos" delante de los participantes". No era cuestión de buscar una solución, al contrario, era cuestión de profundizar y estudiar la situación desde múltiples aspectos. Es importante no sólo buscar soluciones, sino dejar una pausa y dejarlo estar. Necesitamos recordar que en este trabajo aparecen a menudo situaciones ambiguas y otras donde se cuestiona la ética.

Los relatos donde aparecen los dilemas son abiertos en su forma y debemos concentrarnos y sacar a la luz los aspectos que invitan a la reflexión o a la discusión.

\section{ANÁLISIS CUALITATIVOS: TENSIONES SUBYACENTES}

He trabajado fielmente en la tradición del conocimiento práctico y he intentado describir caminos en el terreno. El material, sin embargo, brinda la posibilidad de hacer un análisis cualitativo en temas concretos. Finalmente comentaré algunos aspectos clave encontrados.

Los participantes de los talleres del conocimiento ejercitan su trabajo en el campo social del menor. Durante años, han trabajado sistemáticamente con su desarrollo profesional. Han apostado por la supervisión continuada, la formación postgrado y la organización de proyectos para realizar la colaboración de los usuarios. Les preocupan las redes sociales de apoyo y las formas de comunicación que pueden conseguir un resultado de más igualdad en el equilibrio del poder.

Con este enfoque, podemos hablar de una manera de pensar y un marco de referencia de los profesionales que refleja una reacción ante una tradición donde la actuación del profesional es considerada experta, una tradición que tiene su base en el conocimiento psicológico y en el diagnóstico social, un referente donde el profesional es el que posee el conocimiento.

El material de los talleres del conocimiento nos enseña que los valores como la participación y el enfoque hacia los recursos han sido incorporados como marco de referencia y han influido en la práctica profesional diaria. A la vez, se observa que todavía quedan reminiscencias en un plano más profundo de la manera de pensar de una tradición anterior. Cuando se experimentan situaciones con implicaciones inesperadas como resultado de cambios en la práctica, surgen las inquietudes y las sensaciones de dilema. ¿Hasta dónde podemos trabajar con esta perspectiva en el trabajo social del campo del menor? ¿Hasta qué limites? ¿Cuándo se deben utilizar otros entendimientos?

Es precisamente este tipo de casos límite los que se han expuesto en los talleres del conocimiento.

En los relatos, se ha discutido el valor de la colaboración de los usuarios y cuando este valor ha sido cuestionado. En las preguntas subyacentes se vislumbra la relación entre la protección y la responsabilidad o competencia. En un relato donde se reflexiona sobre cuestiones de colaboración con una madre, 
después de que se ha procedido a tomar la tutela de su hijo, se observa la nueva perspectiva donde aparece el reto de una interpretación del estado psicológico de la madre. Varios de los participantes en el taller piensan que se trata de una madre con una patología psíquica, una madre con necesidad de ayuda. Tal vez, ¿no sería conveniente que el profesional del trabajo social exigiera colaboración de la madre en la situación de trasladar a los hijos a una familia de acogida y que se involucrara personalmente, tomando conciencia de esta situación? Ello podría hacerle más daño psicológico y exponerla a una situación que ella no entiende del todo, ante la cual se ha cerrado emocionalmente.

En contraste con este hecho, se encuentra el punto de vista orientado hacia los recursos, de los que todo ser humano está en su derecho, y debe de tener la posibilidad de tomar una decisión personal. El reto del trabajador social no se encuentra en la valoración de quién es el responsable y quién no se debe de hacer responsable, sino en dialogar de tal manera que la otra parte tenga la sensación de que realmente puede decidir.

Los contenidos de los dilemas en los relatos suelen tratarse, en primer lugar, de relaciones a nivel individual, mientras que en los marcos estructurales del trabajo, como la marginación y la pobreza, se encuentran más periféricos. Este tipo de dilemas no suelen ser temas de discusión en los talleres.

En lo que podríamos calificar como el movimiento entre la práctica profesional reciente y la de antes y en cuanto a conocimientos y competencia, surge el reto de lo que yo llamaría el "entendimiento básico personal".

Surge la pregunta: ¿quién es la persona idónea para comprender las necesidades del niño? Con este tipo de pregunta, sube la temperatura en la discusión de un grupo de profesionales que normalmente trabaja de una manera coherente.

Desde mi postura como investigadora y siguiendo la tradición de la filosofía de la práctica, pienso que en el trabajo social del campo del menor no sólo se necesita un arsenal de relatos y ejemplos sino que se necesita añadir nuevas metáforas que abran la visión a otras maneras de actuar.

Para construir este tipo de metáforas, se puede recoger mucho material en las fuentes antiguas de la sabiduría, y esto se discute en mi trabajo de doctorado (Erstad, 2005).

\section{COMENTARIOS FINALES SOBRE EL PROYECTO LEONARDO}

En este artículo, he querido transmitir las experiencias del taller de los conocimientos como método en un proyecto noruego. Los casos que transmiten los trabajadores sociales tienen su referencia en las discusiones nórdicas sobre la protección del menor. En Noruega, desde hace tiempo, se ha puesto énfasis en los temas que tratan de la participación de los clientes/usuarios y el rol del profesional. En otros países, puede que estos temas sean más periféricos, según los retos con que se encuentran en la problemática del menor.

En el proyecto piloto de Leonardo, se va a transformar un método de investigación hecho en Noruega, hacia un método de formación dirigido a trabaja- 
dores sociales en distintos países europeos. Ello supone un reto a la vez que se vislumbran interesantes posibilidades.

Cada grupo de trabajadores sociales tendrá su propio entrenador/supervisor. Pero son los mismos trabajadores sociales los que van a trabajar con las reflexiones escritas sobre su experiencia personal. Son ellos los que van a elaborar su conocimiento y experiencia. Este tipo de reflexiones, en forma de ensayos, pueden suponer un reto personal, a la vez que es frecuente que se pueda sentir una liberación y una apertura hacia nuevas maneras de ver las cosas. Los masters universitarios que han incorporado el uso de la ensayos como método formativo (Bech-Karlsen, 2003) lo han encontrado positivo. Yo también tengo la misma experiencia actuando como supervisora en otros procesos de expresión por escrito. Lo básico, sin embargo, es que el método requiere siempre la motivación del propio profesional y su deseo de trabajar con la experiencia práctica.

En el taller de los conocimientos, los trabajadores sociales van a compartir sus experiencias. Algunos podrían sentir reparo en contar situaciones donde hubo dudas o dilemas o situaciones donde piensan que actuaban equivocadamente. En mi trabajo de doctorado, el análisis del material me enseñó que los dilemas personales también pueden reflejar un problema que se da en este trabajo en general. Siempre se hace un contrato de colaboración y un compromiso de mantener el secreto profesional. Este acuerdo, de por sí, posibilita el desarrollo de la confianza y apertura en el grupo.

En mi trabajo de doctorado, quería investigar, mediante el diálogo, la comprensión de los trabajadores sociales de su propia práctica. En el análisis del material, encontré lo que habían visto y lo que se encontraba fuera del alcance de ella. Cuando el objetivo de la actividad es el aprendizaje, el supervisor debe sacar a la luz aspectos que pueden complementar las reflexiones y, de ese modo, ampliar la comprensión.

En condición de investigadora y supervisora, he trabajado con mis propias experiencias (Erstad, 1990, 1998) y con grupos de reflexión (Erstad, 1991; Erstad y Saus, 1997). En este proyecto piloto, participan nuevos supervisores que tienen larga experiencia y buenas calificaciones. Sin embargo, necesitan entrenamiento en cuanto al método específico del taller de los conocimientos. Para ello, se ha diseñado un programa especial de formación.

Josefson $(1991,1998)$ discute, con referencia a Aristóteles, cómo la narrativa y otras formas de arte pueden enseñar los aspectos humanos básicos y realmente enriquecer la comprensión profesional. Podemos establecer una analogía entre la visión del artista, por lo singular y la competencia del trabajador a la hora de hacer un juicio profesional de la situación. Aquí hay un reto al talento del profesional de reconocer una situación y a la vez ver lo diferente. En España, se ha iniciado el trabajo de realizar esta actividad utilizando literatura española, estudiando lo tradicional dentro del mundo del arte y las costumbres mediterráneas (Majos, 2006).

El proyecto piloto contiene una dimensión europea que ofrece interesantes posibilidades de intercambio entre países, facilitando dialogos sobre las experiencias y realizando investigaciónes sobre los aspectos semejantes y los 
aspectos diferentes en el ejercicio profesional de la práctica. El objetivo no será necesariamente buscar lo análogo sino llegar a una comprensión de cómo la práctica se basa en tradiciones, cultura, marcos estructurales y formas de entendimiento profesional.

\section{BIBLIOGRAFÍA}

Aristoteles Etikk. Et hovedverk i Aristoteles filosofi, også kalt "Den Nikomakiske etikk". Oversatt og med innledning av Anfinn Stigen. Andre utgave 1996. Gyldendal.

Aristoteles Poetica / Om diktekunsten. Norsk oversettelse fra gresk med innledning og anmerkninger av Sam. Ledsaak. Med et essay om å lese poetikken av Jostein Børtnes. Norsk utgave 1989. Dreyers Forlag A/S.

BECH-KARLSEN, K. (2003). Gode fagtekster. Essayskriving for nybegynnere. Universitetsforlaget.

ERSTAD, I.H. (2005). Erfaringskunnskap og fortellinger i barnevernet. Dr. Polit. Avhandling. Universitetet i Tromsø.

ERSTAD, I.H. (2002). "La investigación en la práctica del Trabajo Social. La experiencia como fuente de conocimientos". Alternativas Cuadernos de Trabajo Social (nº10) 2002. ISSN 1133-0473.

ERSTAD, I.H. (1998). Mellom tradisjon og fornyelse. Studie av en endringsprosess $i$ barnevernet hvor et tradisjonelt barnehjem ble omorganisert til et fylkeskommunalt barne-og ungdomssenter. Hovedfagsoppgave. Universitetet i Tromsø.

ERSTAD, I.(1991). Lederutvikling: Barnevernet inn i90-åra. Rapport fra et lederutviklingsprosjekt. BVU-rapport 3/91.

ERSTAD, I. (1990). Ungdomsbasen i Tromsø. Et tiltak under utvikling. Troms Fylkeskommune.

ERSTAD, I. og SAUS, M. (1997). Samhandling innen barnevernet mellom kommune og fylkeskommune. Rapport 1/97. Barnevernets Utviklingssenter i Nord-Norge.

JANIK, A. (1996). Kunskapsbegreppet i praktisk filosofi. Brutus Östlings Bokförlag Symposion. Stockholm/Stehag.

JOSEFSON, I. (1991). Kunskapens former. Det reflekterade yrkeskunnandet. Carlsons.

JOSEFSON, I. (1998). Läkarens yrkeskunnande. Studentlitteratur. Lund.

LYKKESLET, E. (2003). Bevegelig handlingskunnskap: en studie $i$ sykepleiens praksis. Avhandling dr. polit. Universitetet i Tromsø.

MAJOS, A. (2006). x ref til tittel Upublisert notat $x$

MELØE, J. (1979). Notater $i$ vitenskapsteori for gruppene humaniora og samfunnsvitenskap med fiskerifag. Notat. Universitetet i Tromsø.

MEYER, S. (1995). Pippi leser teksten: essays om språkets makt og maktens språk. Oslo: C. Huitfeldt Forlag.

MOLANDER, B. (1993). Kunskap i handling. Göteborg: Bokförlaget Daidalos.

NERGÅR, J.I. (2000). "Tradisjon og fortelling i samisk medisin". I ALTERN, I, og MINDE, G.T. (red) Samisk folkemedisin i dagens Norge. Skriftserie Nr. 9. Senter for samiske studier. Universitetet $\mathrm{i}$ Tromsø.

NOU 2000: 12. Barnevernet i Norge. Tilstandsvurderinger, nye perspektiver og forslag til reformer. Norges offentlige utredninger.

SAUS, M. (2003). Kontekstuelt barnevern. Barnevern i samiske landskap. Avhandling dr. polit. Universitetet i Tromsø. 